Editorial

\title{
Nutrition in Child Health Conditions
}

\author{
Pilar Codoñer-Franch ${ }^{1,2}$ \\ ${ }^{1}$ Department of Pediatrics, Obstetrics, and Gynecology, University of \\ Valencia, Valencia, Spain \\ 2 Department of Pediatrics, Dr. Peset University Hospital, \\ Valencia, Spain \\ J Child Sci 2018;8:e58-e59.
}

Human health and well-being have advanced substantially during the past two centuries, mainly due to improvements in nutrition, hygiene, and clinical medicine. During this period, a close interrelationship has been established among nutrition, infection, and hygiene. In fact, nutritional status greatly influences infectious disease morbidity and mortality. In most developed countries, improvements in diet have resulted in the virtual disappearance of nearly all nutritional deficiency disorders. However, there is now a transition from nutritional deficiency-related disorders to health problems associated with excessive caloric intake, such as obesity, diabetes, and cardiovascular disease.

In this special issue dedicated to nutrition in child health and disease, we provide an overview of current trends in research and clinical practice focusing on the impact of nutritional status on child health. Nutritional adequacy is defined as the sufficient intake of essential nutrients needed to fulfill requirements for optimal health. The criteria that are now generally used to define adequacy of intake are the prevention of deficiency or chronic diseases and the reduction in risk for diet-associated diseases. Here, we present a review of the special nutritional needs in specific disorders of childhood where diet is essential for effective treatment, such as hypercholesterolemia and nephropathy.

Current and future scenarios also include the shift in importance from under- to overnutrition. Accumulating evidence shows that atherosclerosis begins in youth. The recommended initial step to reduce cholesterol levels in children is the institution of a "heart-healthy" diet, which is safe and often effective. This diet is low in cholesterol and saturated fat and high in complex carbohydrates. It should provide adequate energy for growth, development, and maintenance of a desirable weight.

An appropriately tailored diet is essential for kidney disease patients. Patients with kidney damage should limit the intake of certain foods to reduce the accumulation of unexcreted metabolic products and to protect against hypertension, proteinuria, and other heart- and bone-related health problems.

Address for correspondence

Pilar Codoñer-Franch, MD, PhD, Department of Pediatrics, Obstetrics, and Gynecology,

University of Valencia, Avenida de DOI https://doi.org/

Blasco Ibáñez 15, 46010 Valencia, $10.1055 /$ s-0038-1669378.

Spain (e-mail: pilar.codoner@uv.es). ISSN 2474-5871.
The influences of various nutrients on kidney function and overall health in kidney disease patients has been studied extensively, but there are few studies on the impact of specific diets on patient survival.

Another condition in which diet is an essential aspect of treatment is celiac disease (CD). A strict, lifelong gluten-free diet free of wheat, rye, and barley products remains the treatment of choice for $\mathrm{CD}$. Although the maximum safe amount of gluten for patients with $\mathrm{CD}$ is unclear, $<10 \mathrm{mg}$ daily is probably safe for preventing ongoing intestinal injury. For foods to be labeled gluten-free, there must be $<20 \mathrm{ppm}$. All patients with $C D$ should be referred to a dietitian well-versed in gluten-free diets because parents encounter misinformation and may unnecessarily restrict intake of necessary nutrients. Other practical topics that should be addressed include how to avoid cross-contact at home, travel, and restaurant tips, and how to find reliable information on the Internet.

The review also includes three topics in which diet is of special importance: adolescence, young athletes, and future bone health. Adolescence is generally considered to begin with puberty and to end with completion of physical, mental, and sexual maturation. However, the endpoints are often unclear because adult roles and responsibilities, including the formation of life partnerships and the transition to employment and financial independence, may occur at different ages in different parts of the world, so the cultural concept of adolescence varies greatly.

Adolescent nutrition is crucial for proper growth and development and a prerequisite for achieving full developmental potential. Suboptimal nutrition may contribute to delayed and stunted growth as well as impaired physical and mental development. As adolescents undergo a period of rapid growth and development, adequate intake of both macro- and micronutrients is critical. Eating disorders with different forms of restrictive eating and overweight/obesity are two problems very prevalent at this age.

Nutrition is an integral part of all athletes' lives; however, the importance of proper nutrition is even greater for the

Copyright (C) 2018 Georg Thieme Verlag KG Stuttgart · New York

License terms (๑) $\Theta \circledast$ 
adolescent athlete. Proper nutrition provides all athletes with fuel for energy and the resources necessary to recover from exercise stress. With the rising rate of childhood and adolescent obesity, a great deal of attention is now given to general youth nutrition; however, little focus is given to the specific nutritional needs of the young athlete. In youth athletes, nutrition plays the same vital role as in adult athletes but, more importantly, is also required to support growth and maturation. Young athletes require greater caloric intake than their non-exercising counterparts because of the increased energy expenditures related to training and competition. The greater energy requirements compared with their nonathletic peers include an increased need for protein and, likely, carbohydrate.

Factors that affect bone mineral accrual during growth include genetics, sex, race, diet, mobility, weight-bearing activity, and hormonal factors. The impact of nutrition on bone health has been extensively examined, and various diets, foods, and nutrients have been studied for their potential effects on bone. The effects of dietary components on bone metabolism and bone mass in early life are important because the accretion of bone during growth may help reduce the risk of osteoporosis in later life. The benefits of dairy food products on bone acquisition and maintenance have been the object of particular interest. Among micronutrients, calcium and vitamin $\mathrm{D}$ are especially critical for adequate bone development. However, a significant proportion of children fail to achieve the recommended intake from daily products because of digestive intolerance, so there is emerging recognition of the importance of other animal and/ or plant sources for bone health. The roles of other micronutrients, specifically vitamin $\mathrm{K}$, magnesium, phosphorus, and strontium, also need to be considered regarding their effects on calcium economy, bone metabolism, and resistance to mechanical loading.

Finally, there is emerging interest in the impact of nutrition on circadian rhythms. Melatonin acts as a chemical signal for time of day and its suppression is a marker for disruption of circadian rhythms. Recently, several studies have provided support for a link between altered sleep/wake patterns and obesity, leading to a growing body of research in this area. Children must have sleep of sufficient quality and duration for proper growth and development as growth hormone is mainly secreted at night. Excess evening electric light, whether inside the home or outside in the neighborhood and city, is a rapidly growing problem in the modern world. Prolonged media screen time is now common in children, even preschoolers. The circadian effects could be considerable because computer screens expose children to bright light close to the face, which is the main stimulus for inhibition of melatonin production.

Early identification of how nutrition interacts with child health conditions first requires healthcare providers to be aware of these problems. Qualitative research should be performed to gain insight into how parents can be motivated to emphasize new norms in childhood nutrition. This issue aims to reveal key knowledge gaps that need to be filled to provide stronger dietary advice for the healthcare of children. 